\title{
Relationship of Nanostructured Modulation of Chemical Composition to Magnetic Properties Investigated by HRTEM and Energy Filtered TEM
}

\author{
Ute Golla-Schindler and Andrew Putnis \\ Institute of Mineralogy, D-48149 Münster, Germany
}

The relationship of nanostructure and chemical composition to magnetic properties is of great interest in earth science as well as in material science. In natural rocks remanant magnetisation occurs in various mineral systems, the most important of which are titanomagnetite $\left(\mathrm{Fe}_{3} \mathrm{O}_{4}-\mathrm{Fe}_{2} \mathrm{TiO}_{4}\right)$ and titanohematite $\left(\mathrm{Fe}_{2} \mathrm{O}_{3}-\mathrm{FeTiO}_{3}\right)$ solid solutions. The composition modulation and size of fine precipitates resulting from exsolution generated during cooling control the magnetic properties.

Exsolution in the titanomagnetite solid solution is not possible to study experimentally due to the low solvus temperature $\left(<500^{\circ} \mathrm{C}\right)$ and the slow kinetics. An ideal analogue system is the magnetite $\left(\mathrm{Fe}_{3} \mathrm{O}_{4}\right)$ hercynite $\left(\mathrm{FeAl}_{2} \mathrm{O}_{4}\right)$ system, which has a miscibility gap up to higher temperatures, forming a complete solid solution above $700{ }^{\circ} \mathrm{C}$ [1]. The starting material for the experimental investigations was a series of synthetic samples, quenched from above the solvus under controlled $f\left(\mathrm{O}_{2}\right)$ conditions, with compositions ranging from pure magnetite up to the non magnetic end-member hercynite at compositional intervals of $10 \%$ [2]. The TEM specimens were prepared from a powder both by ion beam milling and crushing under acetone to avoid misinterpretation caused by specimen preparation artefacts. The investigations were performed using the JEOL 3010, a conventional TEM operating at $297 \mathrm{keV}$ equipped with $\mathrm{a} \mathrm{LaB}_{6}$ cathode, a Gatan post column energy filter (GIF) and an Oxford EDX system. All images were gain and dark count corrected and recorded with an attached $1 \mathrm{~K}$ Gatan slowscan CCD Camera.

To determine the magnetic properties we used a Kappa-bridge, which measures the susceptibility as a function of temperature [3]. The heating cycle in the Kappa-bridge induced irreversible changes in the magnetic properties of intermediate compositions of the solid solution. The magnetite -hercynite solid solution has a spinel structure with a face centered cubic unit cell. Spinodal decomposition would be expected in this system in the early stages of unmixing and predicted to occur in the elastically soft $<100>$ directions, leading to three sets of mutually perpendicular modulations. At orientations normal to one modulation, the other two sets of orthogonal modulations form the so-called tweed structure, which is shown in FIG. 1a. The corresponding diffraction image verifies this modulation by showing streaking of the diffraction spots due to the structural modulation FIG.1b. In spinodal decomposition this modulation is also correlated to a sinusoidal spatial variation of the chemical composition. By using element mapping (FIG. 2) we show for the first time the modulation of chemical composition, including the chemical gradients, and hence verify the theoretical expectation. We can quantify the spatially resolved $\mathrm{Fe} / \mathrm{O}$ ratio by mapping the $\mathrm{Fe}$ and $\mathrm{O}$ concentration and using experimentally determined k-factors FIG. 2 b,c,d. We can also calculate the concentration modulation of magnetite and hercynite, which is shown in the linescan FIG. 2 e. The wavelength of the sinusodial modulation and the amplitude offers the possibility of obtaining information about the kinetics of the unmixing mechanism. HRTEM and EFTEM enable us to investigate the evolution of the nanostructure and fluctuations of chemical compositions of interfaces to understand the macroscopic magnetic behavior. [4]

\section{References}

[1] A. C. Turnock and H.P. Eugster Journal of Petrology 3 (1962) 533

[2] H.St. C. O’Neill and A. Navrotsky Am. Mineral. 69 (1984) 733

[3] V. Jelinek and J. Pokorn Phys.Chem. Earth 22 (1997) 179

[4] We thank Dr. S. McEnroe and Dr. H.St. C. O’Neill for the samples and C. Putnis and Dr. B. Schindler for their aid. 

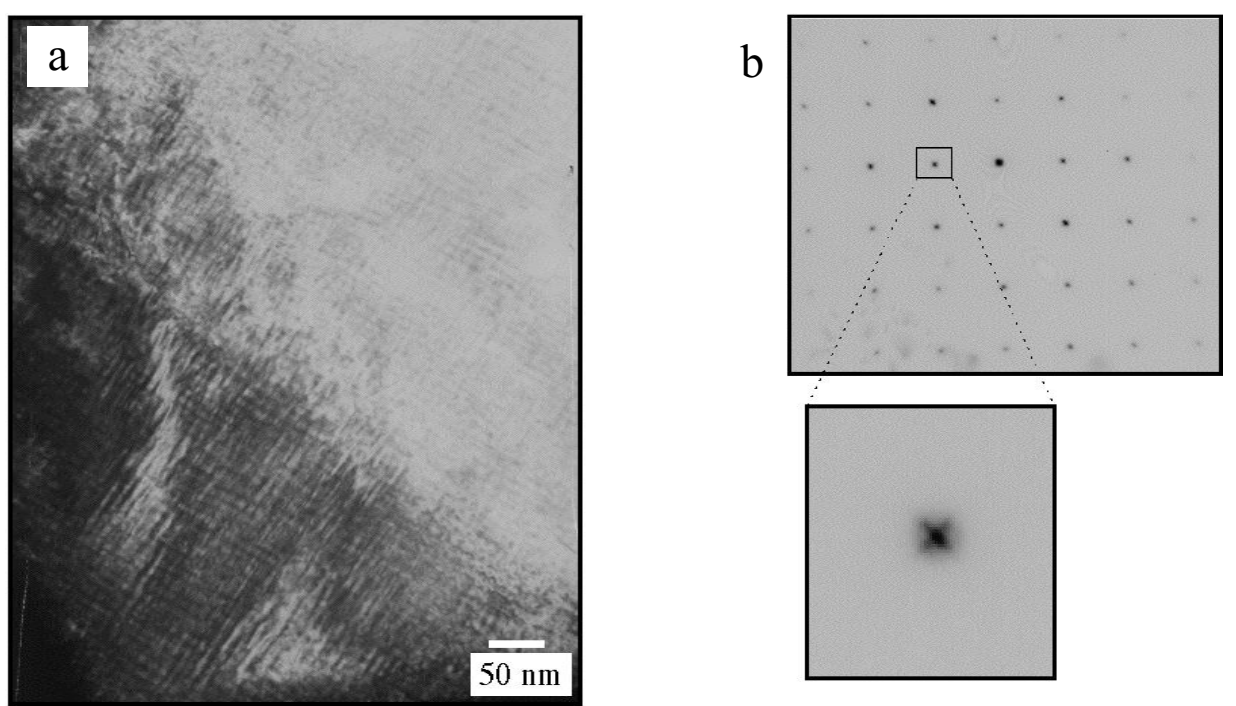

FIG. 1 a, b CTEM image and corresponding diffraction pattern, zone axis [001]. a Tweed structure produced by structure modulation $\mathbf{b}$ corresponding diffraction pattern
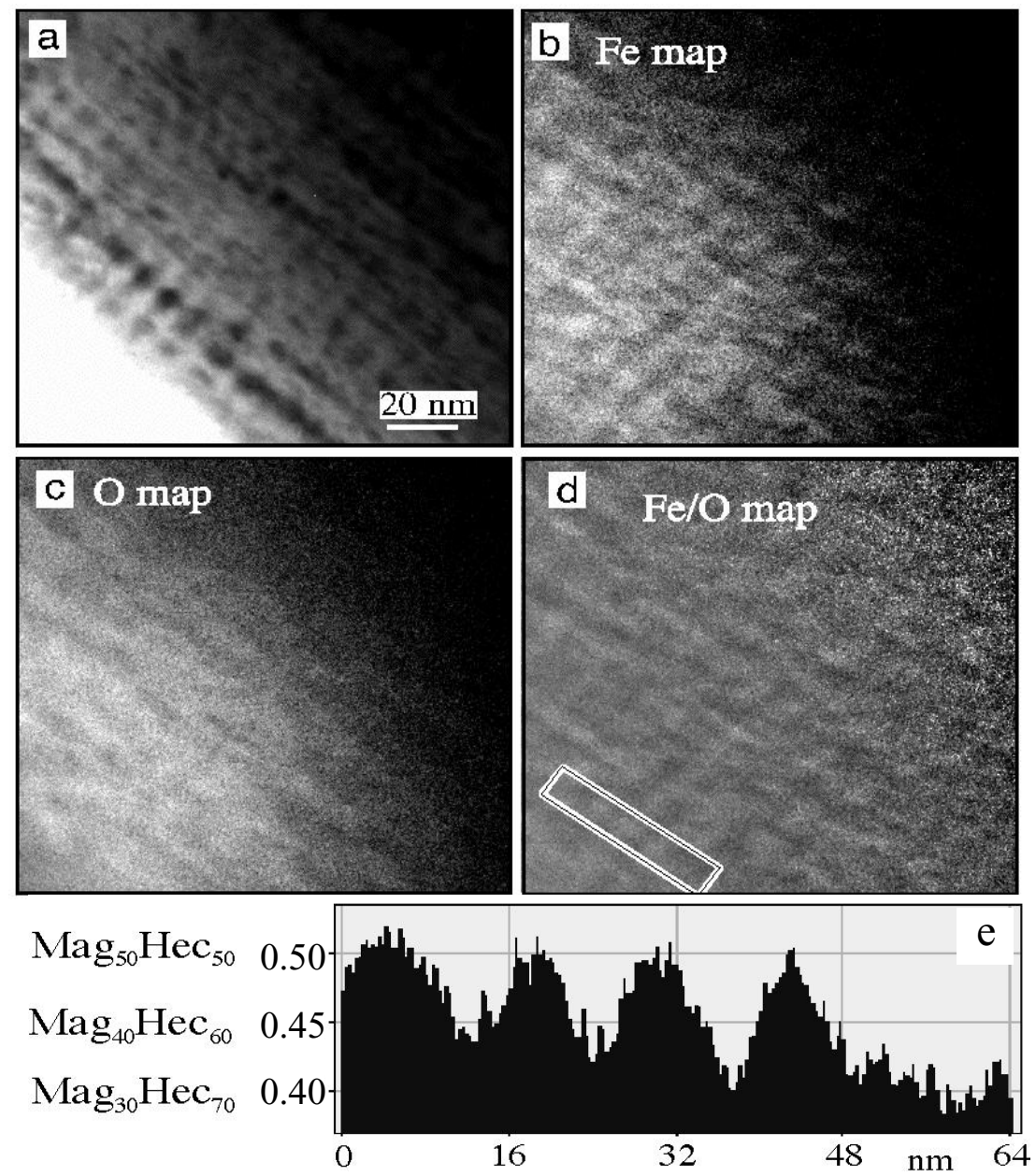

FIG. 2 a-e a CTEM image of spinodal decomposition b,c element map of $\mathrm{Fe}$ and $\mathrm{O}$, respectively $\mathbf{d}$ ratio image of the $\mathrm{Fe} / \mathrm{O}$ element map and $\mathbf{e}$ corresponding linescan of $\mathbf{d}$ 